\title{
Rheological, thermodynamic, and structural studies of linear and branched alkanes under shear
}

\author{
Rajesh Khare ${ }^{\mathrm{a})}$ and Juan de Pablo \\ Department of Chemical Engineering and Rheology Research Center, University of Wisconsin, Madison, \\ Wisconsin 53706
}

Arun Yethiraj

Theoretical Chemistry Institute and Department of Chemistry, University of Wisconsin, Madison, Wisconsin 53706

(Received 2 May 1997; accepted 16 July 1997)

\begin{abstract}
The rheological, thermodynamic, and structural behavior of linear and branched alkanes in simple shear is investigated using nonequilibrium molecular-dynamics simulation of united atom model fluids. Our results for the zero-shear viscosity of pure linear alkanes as well as mixtures of alkanes are in reasonable agreement with experiment. Simulation results for intermediate molecular weight linear alkanes indicate that the simple models employed here are capable of describing the pressure and temperature dependence of the viscosity. More importantly, our calculations indicate that addition of short, flexible branches to alkanes leads to a viscosity enhancement of a factor of 2 or more, thereby offering interesting possibilities for formulation of lubricants with specific properties.

(C) 1997 American Institute of Physics. [S0021-9606(97)50740-9]
\end{abstract}

\section{INTRODUCTION}

Nonequilibrium molecular dynamics (NEMD) simulations provide a useful tool for investigating the rheological behavior of fluids under shear and over the past decade, have been applied to study increasingly complex fluids, including polymers. ${ }^{1,2}$ Such simulations have played, and continue to play, a key role in understanding the relationship between microscopic interactions and macroscopic transport coefficients.

In light of their ubiquity in industrial applications, there has been considerable interest in predicting the rheological properties of alkanes under shear. Edberg et al. ${ }^{3}$ performed NEMD simulations of liquid $n$-butane and $n$-decane using the model of Ryckaert and Bellemans (RB). ${ }^{4}$ These authors found that both alkanes can exhibit a rich nonlinear rheological behavior: shear thinning at relatively small shear rates, normal stresses, and dilatancy. They also studied molecular conformations under shear and found that while shear does not appreciably alter torsional angles in the chain interior, the effect can be significant near the chain ends. This phenomenon was also observed by Brown and Clarke in simulations of liquid $n$-hexane. ${ }^{5}$ Edberg et al. found that the NEMD shear viscosity of liquid $n$-butane at $292 \mathrm{~K}$ and 99 bar was higher than the experimental value by $25 \%$, while the simulated viscosity of liquid $n$-decane at $481 \mathrm{~K}$ and essentially 0 bar was within $6 \%$ of the experimental result. Marechal et al. ${ }^{6}$ obtained results of comparable accuracy for $n$-butane using equilibrium molecular dynamics and a Green-Kubo formalism with a slightly different force field. Chynoweth et al. ${ }^{7}$ performed NEMD simulations on the same model of $n$-butane as Edberg et al. but with 486 molecules, rather than the 64 molecules used by Edberg et al.

\footnotetext{
a) Also at: Theoretical Chemistry Institute and Department of Chemistry, University of Wisconsin, Madison, WI 53706.
}

They obtained a much better resolution of the shear viscosity and found that extrapolations to zero shear gave a Newtonian viscosity in better agreement with experiment.

More recently, the work of Edberg et al. has been revisited by Morriss et al. ${ }^{8}$ These authors conducted simulations of liquid decane under shear using a larger system and extended their calculations to $n$-eicosane. They observed that both decane and $n$-eicosane exhibit a pronounced shearthickening behavior at high shear rates (reduced shear rates $>1.0$ ). Their study also showed that the radius of gyration decreases substantially when the reduced shear rate is greater than 1.0. Unfortunately Morriss et al. used a force field in which nonbonded interactions were purely repulsive, thus clouding the conclusions.

Berker et al. ${ }^{9}$ modified the Ryckaert and Bellemans model by including bond-stretching and angle-bending potentials. They investigated the rheological properties of liquid $n$-hexadecane and were able to observe the first Newtonian regime for the viscosity. However, the Newtonian viscosity predicted by their potential model was a third of the corresponding experimental value. The shear thickening behavior which had previously been observed for liquid $n$-decane and $n$-eicosane by Morriss $\mathrm{et}^{\mathrm{al}}{ }^{8}{ }^{8}$ was not observed by these authors, even at reduced shear rates as high as 6.0. They also compared the strain rate at which NEMD predicted the onset of shear thinning for $n$-hexadecane and found it to be in agreement with predictions of the Rouse model. ${ }^{10}$ They demonstrated that the molecules were clearly deformed as the shear rate increased and postulated that such deformations are responsible for shear thinning.

Although linear alkanes have been the subject of considerable interest, few studies have addressed the effects of molecular architecture, including branching, on their rheological behavior. We believe that research aimed at understanding the effects of molecular geometry on rheological properties 
TABLE I. Alkane models.

\begin{tabular}{|c|c|c|c|}
\hline \multirow{2}{*}{$\begin{array}{c}\text { Physical aspect } \\
\text { Nonbonded } \\
\text { interaction }\end{array}$} & \multirow[t]{2}{*}{ Potential function } & \multicolumn{2}{|c|}{ Parameters } \\
\hline & & $\begin{array}{l}\sigma_{\mathrm{CH}_{3}}=3.93 \AA \\
\sigma_{\mathrm{CH}_{2}}=3.93 \AA\end{array}$ & $\begin{array}{l}\epsilon_{\mathrm{CH}_{3}}=114.0 \mathrm{~K} \\
\epsilon_{\mathrm{CH}_{2}}=47.0 \mathrm{~K}\end{array}$ \\
\hline Bond-bending & $u_{\text {bending }}\left(\theta_{i}\right)=\frac{1}{2} k_{\theta}\left(\theta_{i}-\theta_{\mathrm{eq}}\right)^{2}$ & $\begin{array}{l}\sigma_{\mathrm{CH}}=3.875 \AA \\
k_{\theta}=62500 \mathrm{~K} / \mathrm{rad}^{2} \\
\theta=114^{\circ} \\
\text { linear }\end{array}$ & $\epsilon_{\mathrm{CH}}=31.839 \mathrm{~K}$ \\
\hline Angle torsion & $\begin{array}{c}u_{\text {torsion }}\left(\phi_{i}\right)=V_{0} \\
+1 / 2 V_{1}\left(1+\cos \phi_{i}\right) \\
+1 / 2 V_{2}\left(1-\cos 2 \phi_{i}\right) \\
+1 / 2 V_{3}\left(1+\cos 3 \phi_{i}\right)\end{array}$ & $\begin{array}{l}V_{0}=0 \mathrm{~K} \\
V_{1}=355.03 \mathrm{~K} \\
V_{2}=-68.19 \mathrm{~K} \\
V_{3}=701.32 \mathrm{~K}\end{array}$ & $\begin{array}{l}V_{0}=1357.0 \mathrm{~K} \\
V_{1}=381.73 \mathrm{~K} \\
V_{2}=133.33 \mathrm{~K} \\
V_{3}=-863.8 \mathrm{~K}\end{array}$ \\
\hline Bond stretching & $\begin{aligned} u\left(r_{i j}\right)= & -\frac{H Q_{0}^{2}}{2} \\
& \times \ln \left[1-\left(\frac{r_{i j}-r_{\mathrm{eq}}}{Q_{0}}\right)^{2}\right]\end{aligned}$ & $\begin{array}{l}H=96500 \mathrm{~K} / \AA^{2} \\
r_{\mathrm{eq}}=1.54 \AA\end{array}$ & $Q_{0}=1.572 \AA$ \\
\hline
\end{tabular}

at a molecular level can have important applications in lubrication technology. The ongoing development of metallocene catalysts has facilitated significantly the industrial synthesis of polyolefins of controlled architecture and molecular weight; research of this nature is therefore particularly timely.

Experimental data of Diller and Van Pollen ${ }^{11}$ show that the viscosity of isobutane is much higher than that of $n$-butane over a wide range of density. Rowley and Ely ${ }^{12}$ examined these differences using the RB model and observed the reverse effect, i.e., a higher viscosity for $n$-butane than for isobutane at the same temperature and density. When they increased the size of the methylene groups of isobutane (i.e., $\sigma$ in the Lennard-Jones potential of Table I) by $2.5 \%$, it became possible to obtain quantitative viscositydensity relations over a wide range of densities. In addition, Ely and Rowley also suggested that, for $n$-butane, molecular alignment is responsible for shear thinning at low shear rates. In contrast to their findings for butane, they ${ }^{13}$ found that the origin of the difference in viscosity between liquid $n$-hexane and cyclohexane is primarily due to geometric differences in the molecules.

Daivis et al. ${ }^{14}$ recently studied the rheology of a threearmed "star" alkane (5-butyl nonane), as well as its linear isomer, tridecane. Using a force field in which nonbonded interactions were purely repulsive, they found that branched alkanes exhibit a higher viscosity, weaker shear thinning, and weaker first normal stress differences in the nonlinear region than their linear chain counterparts. At constant volume, the pressure of the branched alkane increased more rapidly with increasing strain rate than that of the linear alkane. These authors suggested that, under shear, the linear molecule elongates and becomes a prolate ellipsoid, while the branched molecule flattens and becomes an oblate ellipsoid. They also observed that the degree of alignment increases less dramatically for the branched molecule than it does for the linear molecule. Overall, Daivis et al. concluded that the flexibility of the molecules is responsible for such differences. We believe, however, that the branched alkane model employed in that work is not entirely satisfactory because apparently the same torsional potential was used for the backbone and for the branches. For torsions around the $\mathrm{CH}-\mathrm{CH}_{2}$ bond at the branching point, however, a different torsional potential that favors gauche conformations, as opposed to trans conformations, is more appropriate.

It is important to point out that viscosity is generally measured with at least one free liquid surface in mechanical equilibrium with the atmosphere, i.e., at constant pressure or at constant normal load, thus allowing the liquid to expand at high shear rates. Recently, Daivis and Evans ${ }^{15}$ have performed isobaric nonequilibrium molecular simulations of decane under shear. They found that the shear-thickening phenomena reported by Edberg et al. in their constant volume simulations disappears. ${ }^{3}$ Other properties, however, such as the shear-induced rotation and the behavior of radius of gyration, are not affected by the change from constant volume to constant pressure. In a similar study, Mundy et al. ${ }^{16}$ used a different force field and reached conclusions analogous to those of Daivis and Evans.

In general, the agreement between simulated and experimental viscosities reported for previous simulations of alkanes has not been entirely satisfactory. Furthermore, depending on the force field employed, different research groups have sometimes arrived at conflicting qualitative trends. Over the past few years better alkane force fields have been reported. Recent results for a limited number of fluids ( $n$-decane) suggest that such force fields are capable of reproducing experimental viscosities over a relatively wide range of pressures. ${ }^{16-19}$ One of the objectives of this work is to investigate whether these force fields can lead to viscosities in good agreement with experiment for a wider range of alkanes over a wider range of temperatures and pressures. Another goal of this work is to investigate the accuracy of such force fields for mixtures of alkanes. To this end, we have measured the viscosity of an alkane mixture and compared it to simulation. More importantly, our work is also 
aimed at increasing our understanding of the relation between branching and rheology for chemically detailed alkane models. Except for the few, noteworthy studies mentioned above, ${ }^{12,14}$ the effects of branching on the rheology of chemically detailed long alkane models have not been examined in detail before. In the present work, results are presented for the rheological properties of branched alkanes under shear and are compared to those of their linear counterparts.

The organization of the paper is as follows. In Sec. II we describe the alkane models and simulation methods employed in this work. In Sec. III we present and discuss our simulation results, and in Sec. IV we present some conclusions.

\section{ALKANE MODELS AND SIMULATION METHOD}

In the united-atom representation of alkanes adopted in this work, every methylene or methyl group of a molecule is modeled as a single interaction site. The models employed in this work are based on original work of Jörgensen et al. ${ }^{20}$ Simple modifications of such potentials have been shown by de Pablo et al. ${ }^{21}$ to provide a reasonable description of vapor-liquid equilibria (VLE) for polyatomic fluids. More recently, in what appear to be the first simulations of phase equilibria for long alkanes (e.g., hexadecane), Laso et al. ${ }^{22}$ showed that it is possible to obtain reasonable agreement with experimental VLE data using a simple alkane model analogous to that of Jörgensen. Siepmann et al. ${ }^{23}$ subsequently modified that model further by fitting orthobaric densities over a wider range of temperatures and chain lengths. That newly reparametrized model provides a good description of saturated densities for linear alkanes, but the corresponding second virial coefficients are in disagreement with experiment. $^{24}$ (Note, however, that more recent calculations suggest that simulated second virial coefficients are in better agreement with experiment than previously reported. ${ }^{25}$ )

The details of the model used in this work are given in Table I. All Lennard-Jones interactions are truncated at $r_{c}$ $=2.5 \sigma$; the potential energy was shifted at the cutoff. Cross interaction parameters are estimated from the usual Berthelot mixing rules, i.e.,

$$
\begin{gathered}
\epsilon_{i j}=\sqrt{\epsilon_{i} \epsilon_{j}}, \\
\sigma_{i j}=\frac{\sigma_{i}+\sigma_{j}}{2},
\end{gathered}
$$

with the exception of the Lennard-Jones parameters for the $\mathrm{CH}$ group of the branched alkane, the linear chain parameters employed in this work are the same as those proposed by Siepmann et al. ${ }^{23}$ The Lennard-Jones parameters for the $\mathrm{CH}$ group employed here are inferred from the values reported by Jörgensen et al. ${ }^{20}$ by requiring that the ratios of $\sigma$ and $\epsilon$ (from Jörgensen to this work) be the same for $\mathrm{CH}_{2}$ and $\mathrm{CH}$ groups. Note that we have not attempted to optimize the Lennard-Jones parameters for $\mathrm{CH}$ groups; this could be done by fitting them to experimental data, but such calculations are beyond the scope of the present study.
In contrast to Jörgensen et al. we employ flexible bonds and flexible bond angles. We have examined the extension of the bonds under shear and we find that the bond length is always within $0.1 \%$ of the desired value of $1.54 \AA$. For bond stretching, Mundy et al. ${ }^{26}$ have employed a harmonic bond potential whose use is questionable for simulations under shear (in the Langevin approximation for dilute, bead-spring chains, it is well known that harmonic springs will stretch indefinitely under the influence of a shear or elongational flow field). In this study, we use a FENE (finitely extendable nonlinear elastic) spring to model bonds between interaction sites. For dilute polymer solutions, FENE springs have been shown to be capable of capturing many experimentally observed rheological phenomena. ${ }^{27}$ Following Chynoweth et $a l^{7}$ bending and torsional forces are determined by reducing such multibody interactions to pseudo-pairwise force terms obeying Newton's third law, i.e., $F_{i j}=-F_{j i}$, where $F_{i j}$ is the force on atom $i$ due to atom $j$. This approach was used by Chynoweth et $a l^{7}$ to develop a parallel algorithm to simulate organic liquids and by Berker et al. ${ }^{9}$ to investigate the rheological behavior of $n$-hexadecane.

The simulations are conducted using SLLOD dynamics. ${ }^{28}$ A velocity Verlet algorithm is used to integrate the equations of motion. Temperature is controlled by means of a Nosé-Hoover thermostat. It is important to bear in mind that isothermal NEMD calculations at extreme shear rates constitute a purely computational strategy for generating results that can subsequently be extrapolated to zero shear. ${ }^{29}$

The flow field imposed on the system corresponds to a planar Couette flow in the $x$ direction with shear along the $y$ axis. For planar Couette flow, the viscometric functions of interest are the apparent shear viscosity $\eta(\dot{\gamma})$, and the first and second normal stress coefficients, $\Psi_{1}(\dot{\gamma})$ and $\Psi_{2}(\dot{\gamma})$, respectively. These can be expressed in terms of the components of the pressure tensor as follows:

$$
\begin{aligned}
& \eta=-P_{x y} / \dot{\gamma}, \\
& \Psi_{1}=-\left(P_{x x}-P_{y y}\right) / \dot{\gamma}^{2}, \\
& \Psi_{2}=-\left(P_{y y}-P_{z z}\right) / \dot{\gamma}^{2} .
\end{aligned}
$$

The atomic pressure tensor $P_{\alpha \beta}$ is evaluated using

$$
P_{\alpha \beta}=\frac{1}{V}\left(\sum_{i} m_{i} v_{i}^{\alpha} v_{j}^{\beta}+\sum_{i} \sum_{j>i} r_{i j}^{\alpha} F_{i j}^{\beta}\right) \text {, }
$$

where $m_{i}, v_{i}, r_{i j}$, and $F_{i j}$ denote the mass of the $i$ th particle, its so-called peculiar velocity with respect to the flow, the distance between particles $i$ and $j$, and the force exerted on particle $i$ by particle $j$, respectively. Superscripts $\alpha$ and $\beta$ denote $x, y$, and $z$ components in the Cartesian coordinate system.

In this work, we investigate the behavior under simple shear of the following alkanes: Linear $\mathrm{C}_{16}, \mathrm{C}_{22}, \mathrm{C}_{28}$, and a branched alkane, namely 5,12-dipropylhexadecane (DPHD). The branches of DPHD are chosen long enough to incorporate the effects of the torsional motion of the side group and are symmetrically positioned along the backbone. 
TABLE II. State points of our NEMD simulations of linear $\mathrm{C}_{16}, \mathrm{C}_{22}$, and $\mathrm{C}_{28}$, and 5,12-dipropyl-hexadecane (DPHD).

\begin{tabular}{lcccc}
\hline \hline & $n-\mathrm{C}_{16}$ & $\mathrm{DPHD}$ & $n-\mathrm{C}_{22}$ & $n-\mathrm{C}_{28}$ \\
\hline Temperature $\left({ }^{\circ} \mathrm{C}\right)$ & 100 & 100 & 100 & 100 \\
Density $\left(\mathrm{g} / \mathrm{cm}^{3}\right)$ & 0.725 & 0.76 & 0.755 & 0.77 \\
Equil. pressure $(\mathrm{atm})$ & $46 \pm 32$ & $62 \pm 35$ & $78 \pm 44$ & $54 \pm 32$ \\
No. of molecules & 100 & 75 & 75 & 80 \\
\hline \hline
\end{tabular}

The state points of our simulations are summarized in Table II for long chain alkanes. The state points are chosen such that at equilibrium (no shear), the pressures of all these fluids are of the order of 50 atm (all of the pressures reported throughout this paper include cutoff corrections). To compare the results of our simulations to literature values, we conducted a few exploratory simulations for linear decane. Our results are identical to those reported by Cui et al. ${ }^{17}$

A cubic simulation box was used in all the calculations. Simulations were carried out over a wide range of shear rates: $2 \times 10^{9}<\dot{\gamma}<2 \times 10^{12} \mathrm{~s}^{-1}$. The time step used to integrate the equations of motion was in general set at (2.35 fs), for which consistent results could be obtained. The duration of the simulations ranged from 2 ns for low shear rates to 565 ps for very high shear rates $\left(\dot{\gamma} \geqslant 10^{12} \mathrm{~s}^{-1}\right)$.

\section{RESULTS AND DISCUSSION}

\section{A. Rheological behavior of alkanes}

Figure 1 shows the viscosity $\eta(\dot{\gamma})$ of the linear alkanes $\mathrm{C}_{16}, \mathrm{C}_{22}$, and $\mathrm{C}_{28}$, and the branched alkane DPHD as a function of shear rate. For clarity, error bars are shown only for the DPHD results. Since experimental data measured at exactly the same conditions of temperature and pressure employed in this work are not available, a number of additional simulations were also conducted at conditions for which reliable experimental data are available. On the one hand, for hexadecane our model alkane at a temperature of $25^{\circ} \mathrm{C}$ and

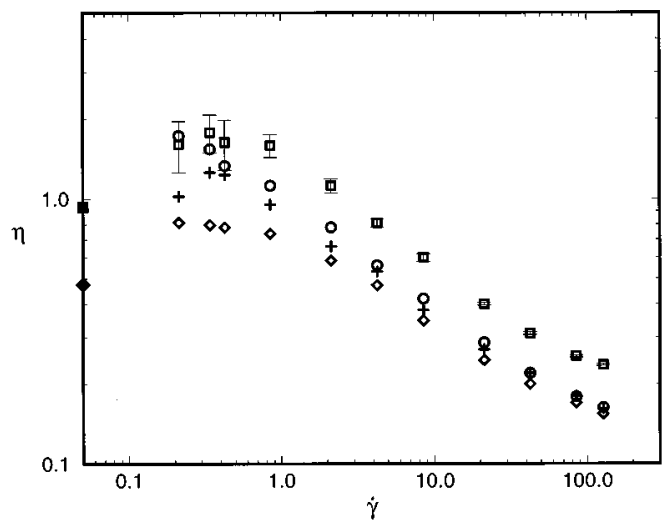

FIG. 1. Shear viscosity $(\mathrm{cP})$ vs shear rate $\left(\times 10^{10} \mathrm{~s}^{-1}\right)$ for linear $\mathrm{C}_{16}$ (diamonds), $\mathrm{C}_{22}$ (crosses), $\mathrm{C}_{28}$ (circles), and a branched alkane DPHD (squares). The symbols on the axis show the zero-shear viscosity of linear $\mathrm{C}_{16}$ calculated using the Assael equation (filled square) and the Lucas equation (filled diamond). density of $0.769 \mathrm{~g} / \mathrm{cc}$ has a pressure of $-13( \pm 28)$ atm and a viscosity of $1.57( \pm 0.33) \mathrm{cP}$. This number for viscosity is consistent with that reported by Cui et al. ${ }^{30}$ who obtained a value of $1.63( \pm 0.28) \mathrm{cP}$ for hexadecane at $27^{\circ} \mathrm{C}$ and a density of $0.770 \mathrm{~g} / \mathrm{cc}$. Cui et al. did not compare their results for hexadecane with experiment; this estimate, however, is below the experimental value of $3.09 \mathrm{cP}$ at $25^{\circ} \mathrm{C}$ at ambient pressure. ${ }^{31}$ For $\mathrm{C}_{28}$, our model alkane at a density of 0.765 $\mathrm{g} / \mathrm{cc}$ and a temperature of $100{ }^{\circ} \mathrm{C}$ has a pressure of 0.4 $( \pm 30)$ atm and a viscosity of $1.62( \pm 0.27) \mathrm{cP}$, in contrast to the experimental value of $2.88 \mathrm{cP}$ at $99^{\circ} \mathrm{C}, 1 \mathrm{~atm}$, and a density of $0.7565 \mathrm{~g} / \mathrm{cc}^{32}$ On the other hand, as stated earlier, our results for viscosity of decane (at $207^{\circ} \mathrm{C}$ and a density of $0.6136 \mathrm{~g} / \mathrm{cc}$ ) are in quantitative agreement with experiments and with the calculations of others. ${ }^{17}$ These results suggest that the alkane model used in this work is capable of reproducing experimental viscosities of low molecular weight alkanes, but the viscosity values obtained for longer alkanes are below the corresponding experimental data. These discrepancies between simulations and experiment could be due to a molecular weight effect or could be due to a density effect. Nevertheless, considering that the alkane parameters employed here were determined from vapor-liquid equilibrium data, this agreement between theory and experiment is somewhat encouraging.

In this study, to ensure that our simulated systems are well in the one phase region, we have chosen to work at slightly elevated pressures which are of the order of $50 \mathrm{~atm}$. There is a lack of experimental data measured at exactly the same conditions of elevated pressure and temperature employed in this work; our simulation results are compared with the values obtained using correlations for viscosity that are commonly used in practice. This comparison is instructive in that we hope that simulations will eventually be used in lieu of correlations when experimental data are not available. The equation developed by Assael et al ${ }^{33}$ (henceforth referred to as the Assael correlation in this paper) is one such widely used correlation. It is derived from fits to extensive sets of experimental data for alkanes over wide ranges of pressure and temperature. Up to relatively high pressures, the viscosity predictions of the Assael equation for linear alkanes are reported to be highly reliable and should therefore be regarded as reasonable measures of true, experimentally determined viscosities. In addition to the Assael equation, we also employ a correlation by Lucas $^{31}$ also based on experimental data. The accuracy of the Lucas equation is also reported to be better than a few percent. The solid symbols in Fig. 1 show estimates of the zero shear-rate viscosity of linear $\mathrm{C}_{16}$ obtained from both Assael and Lucas correlations. As seen from the figure, on the one hand, the plateau value of viscosity for linear $\mathrm{C}_{16}$ is approximately twice the value predicted by the Lucas correlation, ${ }^{34}$ while on the other, it is in very good agreement with that estimated from the Assael correlation. The agreement is particularly encouraging, considering that no attempt was made in this work to adjust the parameters required in the alkane models.

At the lower shear rates investigated here $(\dot{\gamma}<1$ $\left.\times 10^{10} \mathrm{~s}^{-1}\right)$, the viscosities of linear alkanes $\mathrm{C}_{16}$ and $\mathrm{C}_{22}$ 
appear to have reached constant values. On the other hand, at higher shear rates, all the alkanes simulated in this work exhibit shear thinning. At any given shear rate, the simulated viscosity of $\mathrm{C}_{22}$ is consistently bracketed by that of $\mathrm{C}_{16}$ and $\mathrm{C}_{28}$. Similar trends are observed for all other rheological properties investigated in this study, and for clarity, in the remainder of this paper we present results for linear $\mathrm{C}_{16}$ and $\mathrm{C}_{28}$ chains only. The slopes of the linear sections of the graphs are in the range -0.37 to -0.43 for the three linear alkanes. These values of the slopes are slightly below the range of values generally reported for polymeric liquids $(-0.4$ to -0.9$) .{ }^{35}$

The branched alkane DPHD also exhibits shear thinning with an exponent of -0.45 and appears to have reached a plateau value of viscosity at the lower shear rates investigated. The viscosity of DPHD is higher than that of linear $\mathrm{C}_{16}$ (whose backbone chain length is the same as that of DPHD) and $\mathrm{C}_{22}$ (which has the same molecular weight as DPHD) at all shear rates investigated. The results show that branching leads to a viscosity enhancement by approximately a factor of 2 . As mentioned earlier, no attempts were made to optimize the $\mathrm{CH}$ Lennard-Jones parameters employed in this work. To determine the sensitivity of our results to such parameters, we performed two tests for DPHD at a shear rate of $\dot{\gamma}=8.5 \times 10^{9} \mathrm{~s}^{-1}$. In one case, the $\epsilon_{\mathrm{CH}}$ parameter was increased by $20 \%$, and in the other, the $\sigma_{\mathrm{CH}}$ parameter was increased by $1 \%$ over the value reported in Table I. The resulting viscosity values were essentially identical (within statistical uncertainty) to those obtained with the parameters reported in Table I. These findings suggest that the observed viscosity enhancement for DPHD is not a result of our particular choice of parameters but is actually a consequence of chain topology. These results for viscosity enhancement due to short chain branching are consistent with experimental data for medium molecular weight alkanes. Although we could not locate viscosity data for DPHD, experimentally $^{32}$ it is observed that viscosity of 5,14-di$n$-butyloctadecane is approximately twice that of $n$-octadecane at a temperature of $100{ }^{\circ} \mathrm{C}$. The viscosity enhancement observed due to short chain branching in "realistic" chain models used in this work is also consistent with the simulations on fully flexible chain models ${ }^{36}$ where it was observed that zero shear viscosity increased by a factor of 2 when small branches were added to the chain backbone.

At higher shear rates $\left(\dot{\gamma}>10^{12} \mathrm{~s}^{-1}\right)$, the viscosities of all alkanes show a tendency to level off, suggesting the onset of the second Newtonian region. These results, however, should be interpreted with caution because this phenomenon could be entirely due to the thermostat introduced to remove the heat generated by shearing the liquids. As mentioned earlier, recent studies for fully flexible bead-spring chains have shown that, for reduced shear rates higher than about 0.1 $\left(\dot{\gamma}>4.25 \times 10^{10} \mathrm{~s}^{-1}\right.$ ), it is physically impossible to dissipate the viscous heat generated by shearing the fluid fast enough to maintain a constant temperature in the system. ${ }^{29}$

In addition to pure alkanes, we also investigate the rheological behavior of an alkane mixture consisting of $20 \%$ (weight) of normal $\mathrm{C}_{36}$ and $80 \%$ (weight) of normal $\mathrm{C}_{16}$. The

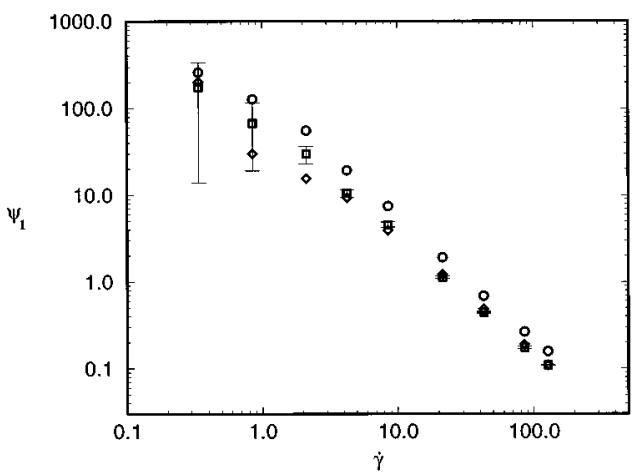

FIG. 2. First normal stress coefficient $\Psi_{1}\left(\times 10^{15} \mathrm{~Pa} \mathrm{~s}^{2}\right)$ vs shear rate $\left(\times 10^{10} \mathrm{~s}^{-1}\right)$ for the branched alkane DPHD (squares), and the linear alkanes $\mathrm{C}_{16}$ (diamonds) and $\mathrm{C}_{28}$ (circles).

density of the mixture was selected to be $0.745 \mathrm{~g} / \mathrm{cm}^{3}$ in our NEMD simulations (for which the system's pressure is equal to the atmospheric pressure, within statistical uncertainty) at a temperature of $80^{\circ} \mathrm{C}$. The simulated zero-shear-rate viscosity of the mixture was found to be $1.05 \pm 0.14 \mathrm{cP}$. Since experimental data for this particular mixture are not available in the literature, we conducted our own measurements of viscosity using a capillary viscometer. The experimental value of viscosity at a temperature of $80^{\circ} \mathrm{C}$ is $1.85 \mathrm{cP}$. Consistent with our results for pure long alkanes, the NEMD estimate is somewhat lower than the experimental value.

In addition to shear thinning, another important characteristic feature of non-Newtonian behavior is the occurrence of normal stresses. Figure 2 shows the variation of the first normal stress difference coefficient $\Psi_{1}$ with shear rate for linear $\mathrm{C}_{16}, \mathrm{C}_{28}$, and the branched alkane DPHD. For clarity, error bars are only shown for DPHD values. We find that $\Psi_{1}$ is consistently positive and exhibits a power-law region, as observed in polymeric fluids. ${ }^{27,35}$ As expected, the rate of decline of $\Psi_{1}$ with $\dot{\gamma}$ is greater than that of $\eta$; the calculated slopes being in the range -1.30 to -1.45 for the linear alkanes. The branched alkane DPHD also exhibits a behavior very similar to that of linear alkanes for the decrease of $\Psi_{1}$ with $\dot{\gamma}$ with a slope of about -1.41 .

For polymeric fluids, the second normal stress difference coefficient $\Psi_{2}$ is negative and its value is much smaller than that of $\Psi_{1}$; the ratio $-\Psi_{2} / \Psi_{1}$ is approximately constant with a value of around 2/7. Figure 3 shows such a ratio for our simulated linear and branched alkanes. Again, error bars are only shown for DPHD. It is observed that for all the alkanes, this ratio is approximately constant in the range 0.4 to 0.5 , with a weak tendency to increase at the higher shear rates. This observed value of the ratio is somewhat higher than that encountered in polymeric liquids, but is consistent with that previously reported in the literature for alkane simulations. ${ }^{9}$

\section{B. Effects of temperature and density on viscosity}

Figure 4 shows the variation of viscosity of $n$-hexadecane with reduced temperature $T_{r}$, defined as $T / T_{c}$ (where $T_{c}$ is the experimental critical temperature). The den- 


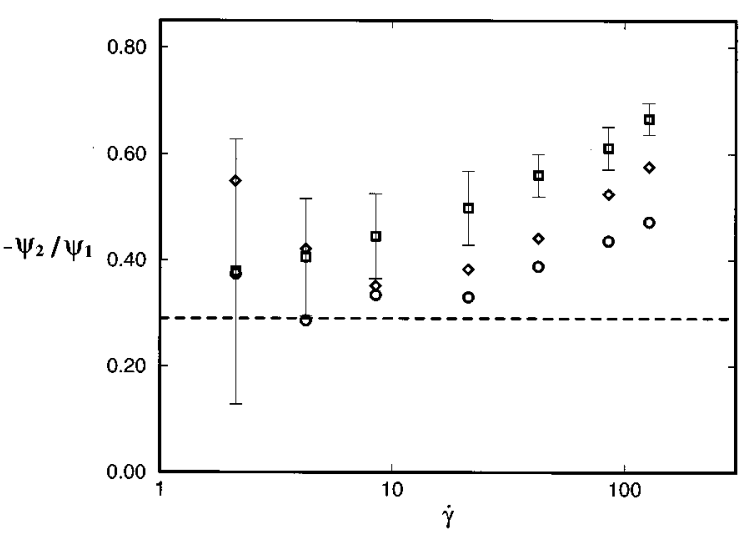

FIG. 3. Ratio of normal stress coefficients: $-\Psi_{2} / \Psi_{1}$ vs shear rate $\left(\times 10^{10} \mathrm{~s}^{-1}\right)$ for the branched alkane DPHD (squares), and the linear alkanes $\mathrm{C}_{16}$ (diamonds) and $\mathrm{C}_{28}$ (circles).

sity of hexadecane at all temperatures was held constant at $\rho=0.725 \mathrm{~g} / \mathrm{cm}^{3}$. The results indicate that for the conditions investigated in this work, the viscosity of linear $\mathrm{C}_{16}$ decreases with rising temperature. The values estimated from the Lucas equation ${ }^{31}$ and from the Assael equation ${ }^{33}$ are also shown in the figure. It is seen that the simulated values are in excellent agreement with the Assael estimates, while the Lucas estimates are about half of the simulated values.

Figure 5 depicts the dependence of the viscosity of $n$-hexadecane on reduced pressure $p_{r}$ at $100{ }^{\circ} \mathrm{C}$. For the range of conditions investigated here, the viscosity of hexadecane increases with pressure (density). Once again the simulation results are in excellent agreement with the Assael correlation, while the values predicted by the Lucas correlation are consistently lower than the simulated values. At the high pressures (densities) investigated in this work, the Assael equation is expected to be more accurate (because high pressure data were used to formulate it) and we therefore view our results as satisfactory.

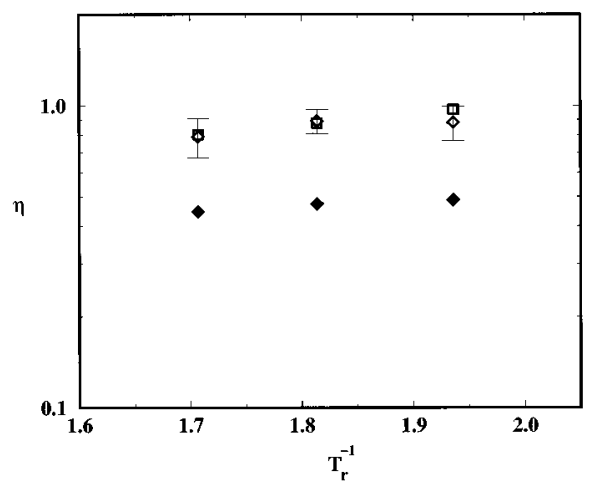

FIG. 4. Viscosity (cP) of liquid $n$-hexadecane as a function of reciprocal reduced temperature $T_{r}^{-1}=T_{c} / T$. Empty diamonds show NEMD simulation results for viscosity. Estimates obtained using the Assael correlation (squares) and the Lucas correlation (filled diamonds) are also shown.

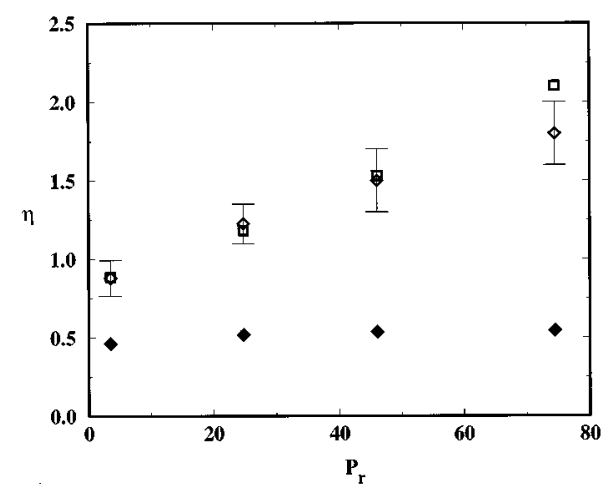

FIG. 5. Viscosity (cP) of liquid $n$-hexadecane as a function of reduced pressure $p_{r}=p / p_{c}$. The figure shows viscosity values obtained using NEMD simulation (empty diamonds), Assael correlation (squares) and the Lucas correlation (filled diamonds).

\section{Thermodynamic properties}

Figure 6 shows the deviation of the system pressure from its value at equilibrium for the linear alkanes $\mathrm{C}_{16}$ and $\mathrm{C}_{28}$ and the branched alkane DPHD. At the lower end of the shear rates investigated here $\left(\dot{\gamma}<5 \times 10^{10} \mathrm{~s}^{-1}\right)$, we find that the hydrostatic pressure of linear alkanes decreases when they are sheared; the magnitude of such a decrease can be of as much as several tens of atmospheres below the equilibrium value. On the other hand, the branched alkane DPHD does not show such a tendency for pressure decrease at lower values of shear rates. At higher values of shear rate, the pressure of both linear and branched alkanes rises rapidly and exhibits a power-law type behavior (as reported previously by Berker et al. ${ }^{9}$ for $n$-hexadecane). A nonmonotonic behavior of pressure as a function of shear rate had been observed earlier in fully flexible bead-spring chains of intermediate length. ${ }^{2}$ Our results for long linear alkanes are consistent with those observations and are discussed further in the context of conformational changes in the following section.

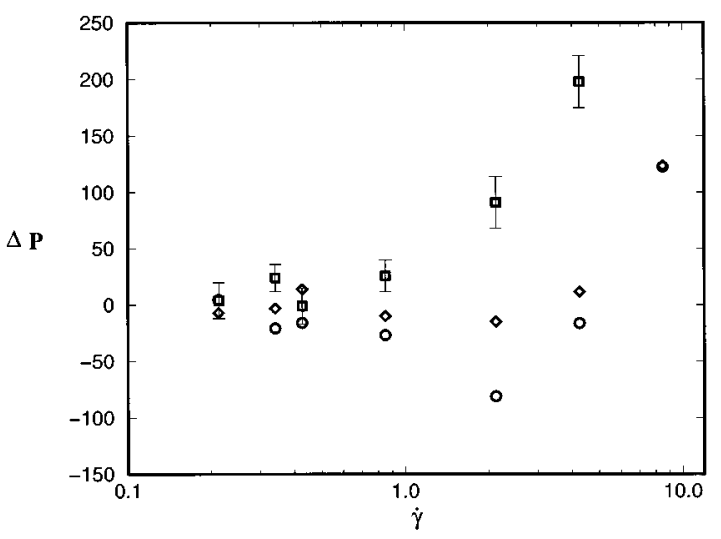

FIG. 6. Hydrostatic pressure change $\Delta P=P(\dot{\gamma})-P(0)$ (atm) vs shear rate $\left(\times 10^{10} \mathrm{~s}^{-1}\right)$ for the branched alkane DPHD (squares), and the linear alkanes $\mathrm{C}_{16}$ (diamonds) and $\mathrm{C}_{28}$ (circles). 


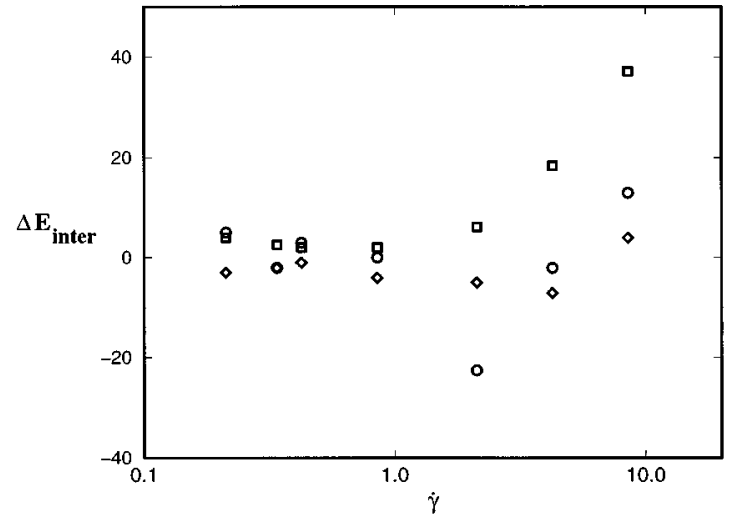

FIG. 7. Change in intermolecular energy $\Delta E_{\text {inter }}=E_{\text {inter }}(\dot{\gamma})-E_{\text {inter }}(0)(\mathrm{kcal} /$ mol) vs shear rate $\left(\times 10^{10} \mathrm{~s}^{-1}\right)$ for the branched alkane DPHD (squares), and the linear alkanes $\mathrm{C}_{16}$ (diamonds) and $\mathrm{C}_{28}$ (circles).

The shear rate dependence of intermolecular part of the energy is similar to that of pressure: It is lower than the equilibrium value at lower shear rates for linear alkanes, while the branched alkane DPHD does not show such a behavior. Figure 7 shows that for shear rates $\dot{\gamma}<5 \times 10^{10} \mathrm{~s}^{-1}$, the intermolecular energy of linear $\mathrm{C}_{16}$ and $\mathrm{C}_{28}$ systems is lower than the equilibrium value by about $5-20 \mathrm{kcal} / \mathrm{mol}$. Similar to $\Delta P, \Delta E_{\text {inter }}$ shows a rapid rise at high values of shear rates for both linear and branched alkanes and also exhibits a "power-law" behavior in agreement with literature results. ${ }^{9}$

\section{Configurational analysis}

In addition to the rheological and thermodynamic properties, we also investigate the configurational properties of long alkanes under shear. In particular, we monitor the shear rate dependence of the chain internal coordinates: bond lengths, bond angles, conformations of the torsional angles, and mean-square radius of gyration $\left\langle R_{g}^{2}\right\rangle$, which provides a measure of chain size. As expected, the average values of $\mathrm{C}-\mathrm{C}$ bond length and $\mathrm{C}-\mathrm{C}-\mathrm{C}$ bending angle hardly change with shear rate, except at extremely high shear rates. A comparison of the fraction of backbone torsional angles in the trans state as a function of shear rate shows that shear flow has a relatively small effect on chain conformation.

Two common parameters used to monitor chain size are the mean square end-to-end distance $\left\langle R^{2}\right\rangle$, and the radius of gyration $\left\langle R_{g}^{2}\right\rangle$. Since $\left\langle R^{2}\right\rangle$ is not well defined for the branched alkane, we only show the variation of $\left\langle R_{g}^{2}\right\rangle$ with shear rate. Figure 8 compares the mean-square radius of gyration of linear $\mathrm{C}_{16}, \mathrm{C}_{22}, \mathrm{C}_{28}$, and the branched alkane DPHD as a function of shear rate. The length of the backbone for DPHD is the same as that of linear $\mathrm{C}_{16}$, but it also has two symmetrically placed branches such that the total number of beads in DPHD is the same as in linear $\mathrm{C}_{22}$. We observe that at the lower values of shear rate, the radii of gyration of $n$-hexadecane and DPHD are almost identical and are significantly lower than that of linear $\mathrm{C}_{22}$. This observation clearly indicates that the branched alkane DPHD,

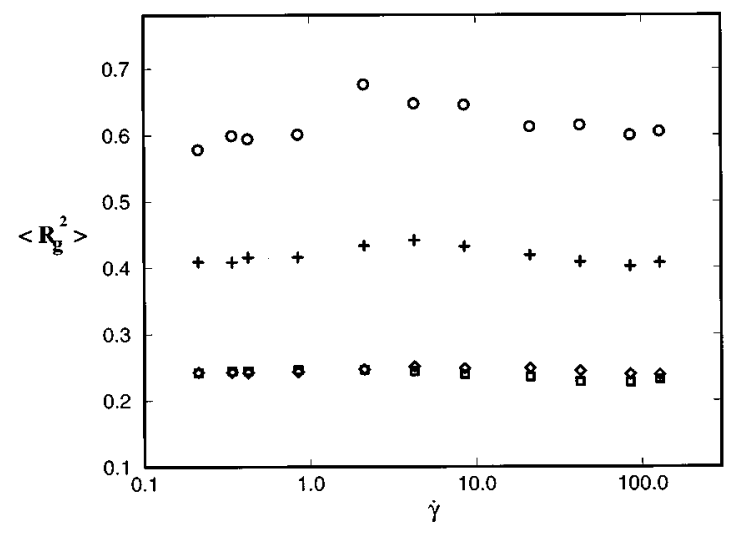

FIG. 8. Average mean-square radius of gyration $\left\langle R_{g}^{2}\right\rangle\left(\mathrm{nm}^{2}\right)$ vs shear rate $\left(\times 10^{10} \mathrm{~s}^{-1}\right)$ for the branched alkane DPHD (squares), and the linear alkanes $\mathrm{C}_{16}$ (diamonds), $\mathrm{C}_{22}$ (crosses), and $\mathrm{C}_{28}$ (circles).

which has branches of realistic flexibility, has a more compact conformation than a linear alkane of comparable molecular weight. Linear alkanes and branched alkanes also exhibit a different dependence of chain size with shear rate: The radius of gyration of long linear alkanes $\left(\mathrm{C}_{22}\right.$ and $\left.\mathrm{C}_{28}\right)$ exhibits a pronounced maximum at intermediate values of shear rates. On the other hand, for the branched alkane, the $\left\langle R_{g}^{2}\right\rangle$ value does not change (within statistical uncertainties) in the range of shear rates investigated. For linear alkanes, the observed maximum in $\left\langle R_{g}^{2}\right\rangle$ with shear rate is consistent with the minimum in pressure and intermolecular energy. At intermediate shear rates molecules are stretched and tend to line up with the flow. At very high shear rates they tumble more frequently and tend to be coiled up. This phenomenon has been reported before for Brownian chains in an anisotropic, hydrodynamic continuum, ${ }^{37}$ for fully flexible chains in a melt, ${ }^{2}$ and for rigid chains in a hydrodynamic continuum in the vicinity of a wall. ${ }^{38}$

\section{CONCLUSIONS}

A united atom model was used to investigate several rheological and thermodynamic properties of linear and branched alkanes under shear. Although results of simulations for the shear viscosity of decane are in good agreement with experiment, the viscosity results for longer alkanes are somewhat below the corresponding experimental values. This behavior suggests that the force field employed in this work is not entirely satisfactory for reproducing the experimental viscosities of longer, liquid alkanes. It is important to note that, at the same temperature and pressure, the density of alkanes increases with chain length. The observed discrepancies could therefore be attributed to chain length effects or density effects. Additional simulations and experimental data are required to determine the origin and exact magnitude of such discrepancies. Nevertheless, considering that the force field employed here was obtained from vapor-liquid equilib- 
rium data, we consider the agreement with experiment to be encouraging. Also note that all experimental trends are reproduced by the simulations.

The effects of temperature and pressure also appear to be described reasonably well by simulations. On the one hand, simulation results are consistent with the predictions of an engineering correlation developed from analysis of highpressure viscosity data for alkanes. On the other hand, our simulated viscosities are consistently higher than those predicted by a different, widely used correlation. These results suggest that, when experimental data are not available, molecular simulations could be used in lieu of correlations. Furthermore, simulations could be used to assess the accuracy of available engineering correlations. Simulations have the important advantage over correlations or group contribution methods of being able to incorporate a detailed account of molecular structure, including branching, and composition. Although our NEMD simulation results for viscosity of an alkane mixture are also in reasonable agreement with experimental data, more calculations for different compositions and more experimental data are clearly needed. Our study should just be viewed as a first step in that direction.

Our results for the hydrostatic pressure of long linear alkanes exhibit a nonmonotonic behavior with shear rate. In the intermediate range of the shear rates studied in this work, the pressure of the systems is well below that observed at equilibrium. At higher shear rates, however, the pressure eventually rises and follows a power-law dependence on shear rate. Long linear alkanes therefore exhibit shear compression at intermediate shear rates and shear dilatancy at extremely high shear rates. These findings are explained mechanistically by discussing the conformational changes undergone by chain molecules in simple shear; similar observations were reported earlier for fully flexible bead-spring chains. ${ }^{2}$ For linear alkanes, the intermolecular energy of the system follows similar trends as the pressure. In contrast, for the branched alkane pressure and intermolecular energy follow monotonic trends with shear rate.

An important finding from our simulations has been that addition of small, flexible branches to linear chains leads to viscosity enhancement by a factor of $\sim 2$. These results are consistent with both experimental data ${ }^{32}$ and our recent simulation results for fully flexible bead-spring chains ${ }^{36}$ where addition of small branches to bead-spring model chains had a similar effect on viscosity. Consistent with earlier findings for star molecules, ${ }^{14}$ we also observe that the conformational behavior of a branched alkane under shear is very different from that of a linear alkane of the same molecular weight.

Although the results of simulations presented in this work are encouraging, particularly in view of their agreement with experiment, significant limitations still exist in the size and timescales that can be investigated by nonequilibrium molecular dynamics simulations. Faster machines and better algorithms will hopefully alleviate some of these problems (e.g., low signal-to-noise ratio at small shear rates) in the coming years.

\section{ACKNOWLEDGMENTS}

The authors thank Professor Ediger for many insightful discussions. This material is based on projects supported by the National Science Foundation (CTS) and by the Petroleum Research Fund of the American Chemical Society. J.J.D.P. is grateful to the $3 \mathrm{M}$ Company, to Xerox, and to DuPont for their support.

${ }^{1}$ M. Kroger, W. Loose, and S. Hess, J. Rheol. 37, 1057 (1993).

${ }^{2}$ Z. Xu, J. J. de Pablo, and S. Kim, J. Chem. Phys. 102, 5836 (1995).

${ }^{3}$ R. Edberg, G. P. Morriss, and D. J. Evans, J. Chem. Phys. 86, 4555 (1987).

${ }^{4}$ J. P. Ryckaert and A. Bellemans, Chem. Phys. Lett. 30, 123 (1975).

${ }^{5}$ D. Brown and J. H. R. Clarke, Chem. Phys. Lett. 98, 579 (1983).

${ }^{6}$ G. Marechal, J. P. Ryckaert, and A. Bellemans, Mol. Phys. 61, 33 (1987).

${ }^{7}$ S. Chynoweth, U. C. Clomp, and Y. Michopoulos, J. Chem. Phys. 95, 3024 (1991)

${ }^{8}$ G. P. Morriss, P. J. Daivis, and D. J. Evans, J. Chem. Phys. 94, 7420 (1991)

${ }^{9}$ A. Berker, S. Chynoweth, U. C. Clomp, and Y. Michopoulos, J. Chem. Soc. Faraday Trans. 88, 1719 (1992).

${ }^{10} \mathrm{M}$. Doi and S. F. Edwards, The Theory of Polymer Dynamics (Clarendon, Oxford, UK, 1986).

${ }^{11}$ D. E. Diller and L. J. Van Pollen, Int. J. Thermophys. 6, 43 (1985).

${ }^{12}$ R. L. Rowley and J. F. Ely, Mol. Phys. 72, 831 (1991).

${ }^{13}$ R. L. Rowley and J. F. Ely, Mol. Phys. 75, 713 (1992).

${ }^{14}$ P. J. Daivis, D. J. Evans, and G. P. Morriss, J. Chem. Phys. 97, 616 (1992).

${ }^{15}$ P. J. Daivis and D. J. Evans, J. Chem. Phys. 101, 541 (1994).

${ }^{16}$ C. J. Mundy, J. I. Siepmann, and M. Klein, J. Chem. Phys. 103, 10192 (1995).

${ }^{17}$ S. T. Cui, P. T. Cummings, and H. D. Cochran, J. Chem. Phys. 104, 255 (1996).

${ }^{18}$ M. Mondello and G. S. Grest (preprint).

${ }^{19}$ C. J. Mundy, M. L. Klein, and J. I. Siepmann, J. Phys. Chem. 100, 16779 (1996).

${ }^{20}$ W. Jorgensen, J. D. Madura, and C. J. Swenson, J. Am. Chem. Soc. 106, 6638 (1984)

${ }^{21}$ J. J. de Pablo, M. Bonnin, and J. M. Prausnitz, Fluid Phase Equilibria 73, 187 (1992).

${ }^{22}$ M. Laso, J. J. de Pablo, and U. W. Suter, J. Chem. Phys. 97, 2817 (1992).

${ }^{23}$ J. I. Siepmann, S. Karaborni, and B. Smit, Nature 30, 330 (1993).

${ }^{24}$ J. Sheng, Ph.D. thesis, Cornell University, 1995.

${ }^{25}$ J. I. Siepmann (personal communication).

${ }^{26}$ C. J. Mundy, J. I. Siepmann, and M. Klein, J. Chem. Phys. 102, 3376 (1995)

${ }^{27}$ R. B. Bird, C. F. Curtiss, R. C. Armstrong, and O. Hassager, Dynamics of Polymeric Liquids, Vol. 2. Kinetic Theory, 2nd ed. (Wiley-Interscience, New York, 1987).

${ }^{28}$ D. J. Evans and G. P. Morriss, Statistical Mechanics of Nonequilibrium Liquids (Academic, New York, 1990).

${ }^{29}$ R. Khare, J. J. de Pablo, and A. Yethiraj, Macromolecules 29, 7190 (1996).

${ }^{30}$ S. T. Cui, S. A. Gupta, P. T. Cummings, and H. D. Cochran, J. Chem. Phys. 105, 1214 (1996).

${ }^{31}$ R. C. Reid, J. M. Prausnitz, and B. E. Poling, The Properties of Gases and Liquids, 4th ed. (McGraw-Hill, New York, 1987).

${ }^{32}$ Properties of Hydrocarbons of High Molecular Weight Synthesized by Research Project 42 of the American Petroleum Institute, New York, 1966.

${ }^{33}$ M. J. Assael, J. H. Dymond, and V. Tselekidou, Int. J. Thermophys. 11, 863 (1990).

${ }^{34}$ The disagreement with the Lucas correlation could be attributed to the value of viscosity of the saturated liquid hexadecane used in this work; this value is required for viscosity calculation using the Lucas correlation. In this work, the viscosity of saturated liquid hexadecane was calculated 
using the Letsou and Stiel correlation from Ref. 31. However, this calculation involves using the correlation just outside of its recommended temperature range of $0.76<T_{r}<0.98$. It is to be noted that if the viscosity of hexadecane at $1 \mathrm{~atm}$ pressure is used in the Lucas correlation (instead of using the saturated liquid viscosity thus calculated), a much better agreement is obtained with both the NEMD simulations and the Assael correlation results.
${ }^{35}$ R. B. Bird, C. F. Curtiss, R. C. Armstrong, and O. Hassager, Dynamics of Polymeric Liquids, Vol. 1. Fluid Mechanics, 2nd ed. (Wiley-Interscience, New York, 1987)

${ }^{36}$ Z. Xu, R. Khare, J. J. de Pablo, and S. Kim, J. Chem. Phys. 106, 8285 (1997).

${ }^{37}$ Z. Xu, J. J. de Pablo, and S. Kim, J. Chem. Phys. 101, 5293 (1994).

${ }^{38}$ J. J. de Pablo, H. C. Ottinger, and Y. Rabin, AIChE J. 38, 273 (1992). 